\title{
Future trends in prostate cancer theranostics with PSMA ligands
}

\author{
Uwe Haberkorn $^{1,2} \cdot$ Klaus Kopka $^{3} \cdot$ Frederik Giesel $^{1,2} \cdot$ Clemens Kratochwil $^{1,2}$
}

Received: 8 April 2016/ Accepted: 2 May 2016/Published online: 14 July 2016

(C) Italian Association of Nuclear Medicine and Molecular Imaging 2016

\section{PSMA as a target for theranostics}

The term theranostics may have different meanings ranging from a very broad to a rather narrow scope of activities. In the broad sense, diagnostics may be used to change therapy planning where the tools used for diagnosis (a radiopharmaceutical) and therapy (radiation therapy, surgery, chemo- or hormone therapy) may be different. In the narrow sense, the same molecule or highly similar molecules are used in vivo for both diagnosis and therapy. This is the case for endoradiotherapy and pre-supposes an appropriate biological target. The attractive feature of endoradiotherapy is that patients may first be identified as possible candidates for this treatment after labeling of the carrier molecule with a $\gamma$ or positron emitter. Upon positive findings, the same molecule can be used for therapy by labeling it with an $\alpha$ or $\beta$-particle emitter. In addition, a pretherapeutic dosimetry is possible. Further advantages of endoradiotherapy over traditional therapies can be expected from the cross-fire effect This effect is induced by the particles originating from the binding site leading to the destruction of multiple cells in the neighborhood of the traceraccumulating cells. The cross-fire effect may compensate to a certain extent for heterogeneous target expression in tumors. This is in contrast to non-radioactive targeting treatment,

Uwe Haberkorn

uwe.haberkorn@med.uni-heidelberg.de

1 Department of Nuclear Medicine, University Hospital Heidelberg and DKFZ Heidelberg, Im Neuenheimer Feld 400, 69120 Heidelberg, Germany

2 Clinical Cooperation Unit Nuclear Medicine, German Cancer Research Center (dkfz), Heidelberg, Germany

3 Division of Radiopharmaceutical Chemistry, German Cancer Research Center (dkfz), Heidelberg, Germany where usually only the cells binding the therapeutic molecule are destroyed. Further enhancement of therapeutic effects is caused by the radiation-induced bystander effect (RIBE). RIBE describes a situation where cells, which have not been directly exposed to the ionizing radiation, behave as if they have been exposed: they die or show chromosomal instabilities or other abnormalities. In this sense, the prostate-specific membrane antigen (PSMA) is an ideal membrane-bound structure for imaging and targeted therapy for prostate cancer: as a transmembrane protein with glutamate-carboxypeptidase activity and its active site located extracellularly it can be addressed by rational design of inhibitors mimicking the binding of the natural substrates, the peptidic neurotransmitter NAAG. Furthermore, after binding of a ligand internalization occurs via clathrin-coated pits and subsequent endocytosis. This results in enhanced uptake and retention in the tumor, prerequisites for a high contrast in imaging and a high local dose deposit obtained in therapeutic applications. Finally, the protein shows high expression in most tumors and low expression in normal tissues. Possible ligands for PSMA are antibodies like J591 and small molecule inhibitors. Both have been used for imaging as well as for therapy and, therefore, are candidates for in vivo theranostics. With respect to the influence of PSMA ligand imaging on the planning of therapies other than endoradiotherapy, several studies showed changes in up to $63 \%$ of the cases in the setting of biochemical relapse and approximately in $25 \%$ in the primary situation [1].

\section{Endoradiotherapy with antibodies and small molecules}

Although phase I and II studies with ${ }^{90} \mathrm{Y}$ - or ${ }^{177} \mathrm{Lu}$-labeled J591 revealed benefits with respect to survival and stabilization of PSA and manageable side effects [2], small 
molecule radiopharmaceuticals have some advantages over antibodies such as better tumor penetration, fast blood clearance and low background activity. This should result in a lower dose for normal organs. Dosimetry with the compound ${ }^{124}$ I-MIP-1095 revealed that the organs receiving the highest absorbed doses after administration of ${ }^{131} \mathrm{I}-$ MIP-1095 are the salivary glands (mean dose $4.6 \mathrm{mGy} /$ $\mathrm{MBq})$, the liver $(1.5 \mathrm{mGy} / \mathrm{MBq})$ and the kidneys (1.5 mGy/MBq). The mean total whole body absorbed dose was $0.38 \mathrm{mGy} / \mathrm{MBq}$ resulting in $0.76-2.7$ Gy based on the injected activities. Doses obtained with ${ }^{177} \mathrm{Lu}-$ PSMA-617 were even lower with $1.4 \mathrm{mGy} / \mathrm{MBq}$ for the salivary glands, $0.1 \mathrm{mGy} / \mathrm{MBq}$ for the liver, $0.75 \mathrm{mGy} /$ $\mathrm{MBq}$ for the kidneys and $0.03 \mathrm{mGy} / \mathrm{MBq}$ for the red marrow. Consequently, side effects with respect to bone marrow were mild to moderate. Diffuse bone marrow involvement was a risk factor for higher grade myelosuppression but could be identified by PSMA-imaging in advance. A therapy response as measured by a decrease in PSA as well as lesion number and size was seen in approximately $70 \%$ for both ${ }^{131} \mathrm{I}$-MIP1095 and ${ }^{177} \mathrm{Lu}-$ PSMA-617 (Fig. 1, [3]). Furthermore, pain relief was obtained in almost all patients.

\section{Future aspects}

A couple of remaining questions have to be addressed for further improvement: which radionuclide has to be used for which situation? In case of endoradiotherapy, should we use $\beta$ or $\alpha$ emitters? According to our preliminary experiences with ${ }^{225} \mathrm{Ac}$ there is a clear benefit of ${ }^{225} \mathrm{Ac}$ over ${ }^{177} \mathrm{Lu}$, especially in patients with disseminated late-stage disease in the bone marrow. This relates to anti-tumor effects as well as to hematologic side effects. However, effects and side effects are a matter of radionuclide, dose and time. Although more efficient with respect to tumor cell killing $\alpha$ emitters will destroy the salivary glands with a reduction of quality of life. For this reason, strategies need to be developed which reduce the tracer accumulation in these organs. Quality of life becomes more important with a longer life expectancy of the patient. This relates to the next question: should we treat early or late or should we even engage in the treatment of minimal disease? Is there a parallel to thyroid cancer? The answer to that question has to take into account potential risks and, as already mentioned, an influence of side effects on the quality of life. Extrapolation from the experiences obtained from Nagasaki and Hiroshima and the nuclear plant accident in Tchernobyl reveals a theoretically increased risk of 2-3\% for leucemia at 5-15 years after treatment. Furthermore, a theoretical risk for a decrease in kidney function after 5 years has to be considered. These theoretical risks as well as a possible xerostomia are not an issue in the final stage patient, but could be problematic in patients treated earlier. Therefore, a decision for early treatment should rely on the identification of high-risk patients, but at present it is questionable that the criteria needed for such a decision are in our hands.

In addition, we may ask whether a monotherapy with PSMA ligands is really the end of the story? Since PSMA expression may be enhanced by androgen-deprivation therapy a premedication with antiandrogen drugs may increase the tracer accumulation and consequently the dose in tumor lesions. Another aspect to be considered is the obvious heterogeneity of PSMA expression in primary tumors and metastases. This heterogeneity may lead to the selection of clones with low or even no PSMA expression and, therefore, to resistance to endoradiotherapy with PSMA ligands. In the latter case, new biological targets
Fig. 1 A patient with metastasized prostate cancer. Pretherapeutic PET/CT with ${ }^{68}$ Ga-PSMA-11 (left) and intratherapeutic scintigraphy during the first, second and third application of ${ }^{177}$ Lu-PSMA617. During the course of PSMA ligand therapy, the number of tumor lesions decreased which was accompanied by a decrease in PSA

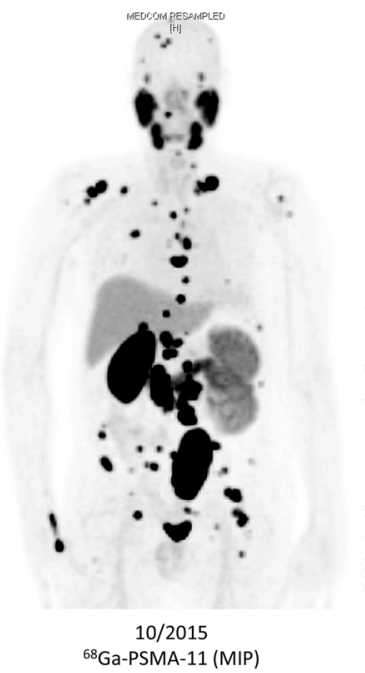

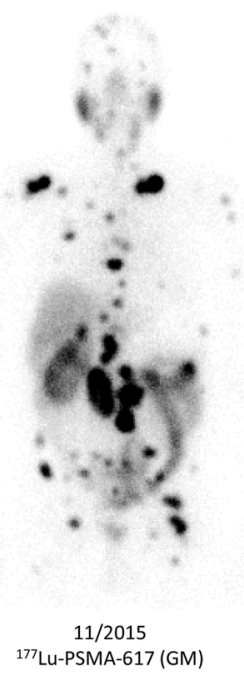
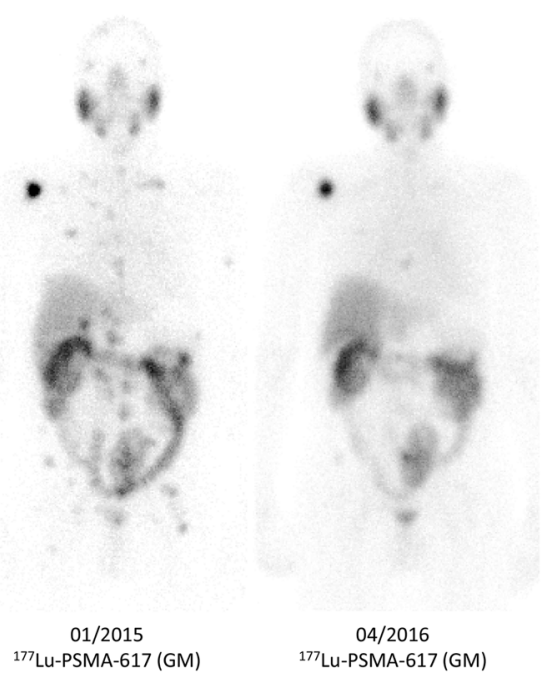
and new radiopharmaceuticals have to be identified and developed for clinical application.

\section{Compliance with ethical standards}

Conflict of interest All authors declare that they have no conflict of interest.

Ethical approval This article does not contain any studies with human participants performed by any of the authors.

\section{References}

1. Haberkorn U, Eder M, Kopka K, Babich JW, Eisenhut M (2016) New strategies in prostate cancer: prostate- specific membrane antigen (PSMA) ligands for diagnosis and therapy. Clin Cancer Res 22:9-15

2. Bander NH, Milowsky MI, Nanus DM, Kostakoglu L, Vallabhajosula S, Goldsmith SJ (2005) Phase I trial of ${ }^{177}$ Lutetium-labeled J591, a monoclonal antibody to prostate-specific membrane antigen, in patients with androgen-independent prostate cancer. J Clin Oncol 23:4591-4601

3. Kratochwil C, Giesel FL, Stefanova M, Benešová M, Bronzel M, Afshar-Oromieh A, Mier W, Eder M, Kopka K, Haberkorn U (2016) PSMA-targeted radionuclide therapy of metastatic castration-resistant prostate cancer with Lu-177 labeled PSMA-617. J Nucl Med. pii: jnumed.115.171397 [Epub ahead of print] 